\title{
Trastornos del Lenguaje en el Adulto
}

\author{
Language disorders in adults
}

\section{Dr. Archibaldo Donoso S.†}

Neurólogo

Dpto. Neurología-Neurocirugía Hospital Clínico

Universidad de Chile

\section{Prof. Rafael González V.}

Fonoaudiólogo

Dpto. Neurología-Neurocirugía Hospital Clínico Universidad de Chile

\section{RESUMEN}

El presente trabajo tiene por objetivo principal describir los trastornos del lenguaje en el adulto a consecuencia de un daño cerebral. Las alteraciones del lenguaje oral se abordarán considerando los siguientes tópicos: Algunos conceptos lingüísticos básicos para describir con exactitud las alteraciones del lenguaje; el sistema funcional del lenguaje en el que participan áreas persilvianas, hemisferio derecho y zonas prefrontales; las afasias que son un trastorno parcial o total del lenguaje; los pacientes afásicos considerando sus antecedentes clínicos, la actitud frente al defecto, el deterioro intelectual y otros defectos asociados; el tratamiento de las afasias que tiene por objetivo que el paciente logre una comunicación funcional y una adaptación psicosocial en relación a la alteración de lenguaje y por último, los trastornos del discurso que comprometen especialmente su contenido y su uso. En relación a las alteraciones del lenguaje lectoescrito se describirán los diferentes tipos de agrafias y alexias.

Finalmente, cabe señalar dos aspectos, el primero, es que además de las afasias existen otros trastornos del lenguaje y el segundo, es que la evaluación del lenguaje es fundamental para el manejo y la rehabilitación del paciente.

Palabras claves: afasia, paciente afásico, tratamiento de la afasia, trastornos del discurso, trastornos de la lectoescritura.

\section{ABSTRACT}

The main purpose of the current review is to describe the language disorders in adult as a consequence of brain damage. The oral language disorders will be approached regarding the following topics: some basic linguistic concepts to describe language disturbances accurately; the functional language system involving perisylvian areas, right hemisphere and prefrontal areas; aphasia that represent a total or partial language impairment; the patient with aphasia, regarding their clinical background, their attitude towards the alteration, the cognitive impairment and other associated disorders; the aphasia treatment which has for purposes to achieve a functional communication and psychological and social adaptation considering their language impairment and, at last, the discourse impairment which affects their use and content. Regarding the reading and writing impairment, agraphia and alexia types will be described.

Finally, it is important to mention two aspects: first, besides aphasia there are other language disorders and, second, language assessment is essential to the management and rehabilitation of the patient.

Key words: aphasia, aphasic patient, aphasia treatment, discourse impairment, writing and reading impairment.
Contacto con el autor: Rafael González V. Depto. Neurología-Neurocirugía, Hospital Clínico Universidad de Chile. Santos Dumont 999 Santiago-Chile. Tel: (56 2) 7773882 Correo-e: ragonvic@gmail.com 


\section{Introducción}

Las funciones cerebrales superiores (FCS) incluyen el lenguaje, la memoria, la percepción visual, capacidades práxicas y la capacidad ejecutiva entre otras. Están organizadas como sistemas funcionales y dependen de una constelación de áreas corticales y subcorticales (y sus conexiones), cada una de las cuales hace una contribución relativamente específica al funcionamiento normal ${ }^{1}$. Este concepto de Luria es similar al concepto contemporáneo de redes neuronales. Hace años se postulaba que dependían exclusivamente de la corteza cerebral, pero en las últimas dos décadas se ha demostrado la importante contribución de las estructuras subcorticales; aunque aun se discute si la participación de estas estructuras es directa o indirecta $^{2,3,4}$.

La evaluación de estas funciones siempre ha puesto a prueba la habilidad clínica de los neurólogos y fonoaudiólogos, debido a su compleja fisiología que hace que los rendimientos de los pacientes con una lesión cerebral de igual topografía puedan ser diferentes, con variaciones incluso en un mismo sujeto de un momento a otro. Además influyen la imprecisión de algunos conceptos y las dificultades para describir o cuantificar las deficiencias. A modo de ejemplos, podemos decir que no siempre las lesiones frontales determinan un síndrome frontal, o que la capacidad de comunicarse de un paciente afásico puede cambiar según el contexto. Además diversos autores han definido en forma diferente lo que es una agrafia; y es necesario saber algo de lingüística para describir con precisión los trastornos del discurso.

En esta revisión se describirán las consecuencias que tienen las lesiones cerebrales en el lenguaje oral y la lectoescritura.

1. Trastornos del lenguaje oral.

Se revisaran algunos conceptos lingüísticos básicos, el sistema funcional del lenguaje, las afasias, algunas características de los pacientes afásicos, su tratamiento, y los trastornos del discurso.

\subsection{Conceptos lingüísticos básicos.}

En el lenguaje normal se distinguen el contenido, la forma y el uso ${ }^{5}$. El contenido se refiere a los conceptos, al pensamiento que se traduce en lenguaje expresivo o que se deduce al escuchar al interlocutor. Se puede alterar en psicosis lúcidas como la esquizofrenia (con trastornos del curso formal del pensamiento), en estados de confusión mental y en demencias.

La forma se refiere a los niveles fonémico, morfosintáctico y léxico, a la correcta selección de fonemas, morfemas, gramemas y al uso de las reglas de combinación. En los sujetos diestros depende especialmente de las áreas perisilvianas izquierdas. Los fonemas, a nivel segmentario, son partículas que pueden hacer cambiar el significado de una palabra. Por ejemplo /m/ y /p/ son fonemas porque si en la palabra /mesa/ el primer fonema se cambia por /p/ cambia el significado de la palabra. El nivel suprasegmentario de los fonemas se refiere a la prosodia, a la melodía de las frases. 
Los morfemas están constituidos por uno o más fonemas y son partículas mínimas con significado; por ejemplo el morfema <camp> significa relativo al campo y se puede usar en las palabras campo, acampar, campesino, etc. El morfema <o> significa género masculino y está constituido por un solo fonema. En las afasias por lesión del área de Wernicke son características las parafasias, esto es palabras erróneas, en que pueden sustituirse los fonemas (parafasia fonémica, por ejemplo /fása/ por /kása/) o los morfemas (parafasia semántica, por ejemplo mesa por silla). En las parafasias verbales, en que se sustituye una palabra por otra sin relación evidente (casa por fósforo), y en los neologismos afásicos (/koríne/ por /árbol/) no se logra adivinar la relación entre la palabra correcta y la errónea . Los gramemas son morfemas con significado gramatical: /os/ significa género masculino y plural, /a/ femenino y singular, la conjunción /y/ significa cierta relación asociativa, /rá/ un tiempo futuro. En una frase bien construida se respetan las reglas de combinación de morfemas básicos y gramemas: "los niños juegan" es correcto, "los niños jugar" es incorrecto, sería un ejemplo de agramatismo.

El uso del lenguaje se refiere al respeto a las reglas del diálogo: la alternancia en los roles de emisor y receptor, considerando el contexto (suponer lo que sabe el otro) para ser más o menos explícitos, el intentar que el mensaje sea recibido dirigiéndonos al interlocutor en forma adecuada (no es lo mismo una autoridad que un compañero de curso). Este uso del lenguaje (o pragmática) puede alterarse por lesiones del hemisferio derecho, cuando la negligencia unilateral hace que el paciente se dirija hacia un costado y no al examinador; en un delirio agudo cuando el paciente imagina que el examinador es un familiar; en los síndromes prefrontales. En las afasias se altera especialmente la forma del lenguaje; en los trastornos del discurso se hacen más evidentes las alteraciones del contenido y del uso.

\subsection{Sistema funcional del lenguaje.}

En el sistema funcional del lenguaje participan áreas perisilvianas izquierdas, estructuras subcorticales, el hemisferio derecho y las áreas prefrontales $^{6}$. Esto ha sido comprobado en pacientes con lesiones cerebrales focales y en voluntarios normales.

La afasia amnésica, en la cual existe una disociación del signo lingüístico, entre el significado y el significante, puede deberse a lesiones frontales, frontoestriatales, temporales o parietales. Se han descrito pacientes con defectos específicos en la denominación de personas, animales o herramientas, lo que es concordante con la activación de diferentes áreas corticales cuando los sujetos normales denominan miembros de esas categorías ${ }^{7}$.

En el área de Broca existen subunidades que tienen a su cargo el control de la articulación y de la prosodia. Por este motivo las lesiones del área de Broca pueden causar apraxia del habla (antes llamada disartria cortical) y pérdida de prosodia. Trabajos de la última década han demostrado que las lesiones de la corteza insular anterior izquierda son responsables de la apraxia del habla ${ }^{8}$. Otra subunidad del área de Broca es el sustrato de la gramática, de las reglas de combinación que constituyen la morfosintaxis. Así se explica que en las afasias de Broca existan 
combinaciones variables de apraxia del habla, aprosodia y agramatismo ${ }^{9,10}$.

El área de Wernicke es importante para el lenguaje expresivo y para la comprensión del lenguaje. Con respecto al lenguaje expresivo permite la selección de los morfemas básicos (la selección de los fonemas dependería principalmente de la circunvolución supramarginal). Por otra parte, constituye una conexión indispensable entre las áreas auditivas y el resto del cerebro, a través de las circunvoluciones angular y supramarginal. La primera es un área de asociación terciaria que permite asociar sonidos con imágenes visuales o somestésicas, atribuir un significado a lo que escuchamos. Al hablar, permite encontrar el significante del concepto que tenemos en mente. La circunvolución supramarginal, junto al fascículo arcuato, es un área decisiva en el procesamiento fonológico. Esto explica que los afásicos de Wernicke tengan en su lenguaje expresivo parafasias fonémicas, semánticas o de otros tipos, y que presenten defectos en la comprensión auditiva ${ }^{10}$. Por otra parte, en las afasias de conducción con compromiso del fascículo arcuato y/o la corteza de la circunvolución supramarginal; existen parafasias fonológicas, que el mismo paciente detecta e intenta corregir.

Existe un circuito entre el área de Wernicke, el tálamo óptico y el área de Broca. Según Crosson este circuito sería fundamental para el monitoreo semántico del enunciado, y por eso las lesiones talámicas pueden causar una afasia transcortical sensorial con abundantes parafasias semánticas. Por otra parte, el cuerpo estriado parece importante para el inicio y la fluidez del discurso ${ }^{11}$.
El hemisferio derecho también participa en el lenguaje. Cuando un paciente afásico comienza a recuperar el lenguaje, esta mejoría depende, por lo menos en parte, de la capacidad del hemisferio derecho para hacerse cargo de las funciones perdidas, lo que se ha comprobado con neuroimágenes funcionales. Un caso extremo es el lenguaje en sujetos que han sido sometidos a hemisferectomía izquierda ${ }^{12}$. Por otra parte, las lesiones del hemisferio derecho pueden causar pérdida de prosodia, dificultades para interpretar metáforas, trastornos de la coherencia del discurso y fallas en la selección de los detalles significativos de un relato ${ }^{13}$.

Las áreas prefrontales son importantes para la programación, regulación y verificación de la conducta $^{1}$. Esto también rige para el lenguaje, y las lesiones prefrontales, especialmente si son izquierdas, ya que pueden alterar el discurso con laconismo, faltas de coherencia y perseveraciones haciéndolo menos informativo que en los afásicos ${ }^{14}$. Las lesiones frontales derechas pueden causar pérdida de prosodia y de coherencia. En el párrafo destinado al discurso analizaremos con más detalle estos conceptos.

A modo de ejemplo de lo que es el sistema funcional del lenguaje, hemos revisado los trastornos de la denominación ${ }^{6}$. La denominación normal involucra varias etapas: la disposición a la tarea, tener una clara imagen mental del referente, seleccionar el morfema básico y los gramemas adecuados, elegir los fonemas que componen esos morfemas, articular esos fonemas. Por lo tanto, puede alterarse a varios niveles: un paciente 
psicótico puede negarse a rendir, un demente puede perseverar en las palabras previamente usadas, un paciente con agnosia visual o una demencia semántica no reconoce el objeto ni evoca sus atributos, un afásico puede presentar anomia o parafasias, un paciente con disartria puede emitir fonemas irreconocibles. Por otra parte, sabemos que existen anomias específicas para personas, para animales y para herramientas ${ }^{7}$. Ello dependería de las redes neuronales que subyacen al conocimiento semántico de esas diferentes categorías de objetos.

\subsection{Las afasias.}

Las afasias pueden ser definidas como un trastorno parcial o total de la forma del lenguaje. Se deben a una lesión de las áreas de asociación del hemisferio dominante para el lenguaje, que en la mayoría de los diestros es el izquierdo ${ }^{9,10}$. El defecto nuclear de las afasias es el defecto en la denominación, la disociación del signo lingüístico, entre el significado (el concepto, que la mayoría de los pacientes conserva) y el significante (la palabra que lo representa, de la cual no se dispone). La anomia, esto es la dificultad para encontrar palabras es muy sensible a la frecuencia de la palabra: es más difícil evocar palabras infrecuentes que las de uso cotidiano. Una característica que comparten las afasias con otros defectos en las FCS es la diferencia entre el rendimiento proposicional, voluntario, y el rendimiento automático, emocional: la expresión verbal es mejor en un ambiente natural que en una sala de examen, es mejor cuando nace de los deseos del paciente y no de las exigencias del clínico.
Para estudiar las afasias podemos conversar con el paciente, observando si en sus respuestas existe o no una relación normal entre fluidez e información, si existen dificultades o errores en la selección de los sustantivos, deformaciones fonológicas o errores articulatorios, si se respetan o no las reglas de la gramática. Si el sujeto tiene dificultad para encontrar los nombres y presenta parafasias será más fluente que informativo; esto sugiere compromiso de las áreas posteriores del lenguaje, por detrás de la cisura de Rolando. Si hay laconismo, si el sujeto es más informativo que fluente, es probable que la lesión sea prerrolándica. También debe estudiarse la denominación, la descripción de una escena y la comprensión. Con respecto a esta última es necesario observar si las respuestas son 0 no atingentes, si cumple instrucciones de complejidad variable (saque la lengua; saque la lengua y tóquese la nariz con la mano izquierda; señale cuál escena corresponde a la frase "el perro es perseguido por el niño" etc.). La repetición es importante para diagnosticar las afasias de conducción y transcorticales.

La clasificación de las afasias es compleja; además debe aceptarse que en muchas ocasiones no se logra clasificar con facilidad a algunos pacientes o en ellos no se observa la correlación anátomo-clínica que podría esperarse ${ }^{15}$. En esta sección se revisa la clasificación de Goodglass y Kaplan ${ }^{10}$. Esta escuela reconoce las afasias de Broca, de Wernicke, de conducción, anómica, las afasias transcorticales, la afasia mixta anterior, la afasia global y defectos aislados del lenguaje (afasia táctil, alexias y agrafias). En la tabla 1 se resumen sus características. 
Tabla 1. Características principales de las afasias.

\begin{tabular}{|c|c|c|c|c|c|c|c|c|}
\hline & Anómica & Wernicke & Broca & Conducción & $\begin{array}{c}\text { Transcortical } \\
\text { motora }\end{array}$ & $\begin{array}{c}\text { Transcortical } \\
\text { sensorial }\end{array}$ & $\begin{array}{l}\text { No fluente } \\
\text { mixta }\end{array}$ & Global \\
\hline $\begin{array}{l}\text { Pérdida } \\
\text { fluidez }\end{array}$ & - & - & ++ & + & ++ & 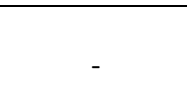 & ++ & ++ \\
\hline Aprosodia & - & - & ++ & + & + & - & ++ & ++ \\
\hline Anomia & ++ & ++ & + & + & - & + & ++ & ++ \\
\hline Agramatismo & - & - & ++ & + & - & - & ++ & ++ \\
\hline Parafasias & - & ++ & - & ++ & - & ++ & + & ++ \\
\hline $\begin{array}{l}\text { Déficit } \\
\text { repetición } \\
\text { Déficit } \\
\text { comprensión }\end{array}$ & - & ++ & + & ++ & - & - & ++ & $\begin{array}{l}++ \\
++\end{array}$ \\
\hline
\end{tabular}

La afasia anómica o amnésica se observa en pacientes con lesiones pequeñas del lóbulo temporal, parietal o frontal. Se caracteriza por el defecto en denominar objetos, en evocar el morfema básico, los sustantivos. Cuando un paciente afásico comete errores para denominar puede ser por un defecto del léxico o por un defecto semántico ${ }^{16}$. En el primer caso falla en la denominación pero maneja el concepto, puede establecer asociaciones semánticas entre ese y otros objetos, puede seleccionar el nombre correcto entre varios. En el segundo caso carece del concepto, no es capaz de señalar las propiedades ni asociaciones del objeto con otros objetos ni con su nombre correcto, se conduce como un agnósico. El defecto léxico puede ser considerado una amnesia procedural verbal. Generalmente se asocia a fallas en pruebas de fluidez verbal (o memoria semántica: enumerar ítems dentro de una categoría, por ejemplo animales) y de aprendizaje de series de palabras. La articulación, la prosodia, el uso de la gramática, la repetición y la comprensión son normales y la severidad del defecto en la comunicación es discreta.
La afasia de Broca se caracteriza por la apraxia del habla, pérdida de prosodia y agramatismo. En la apraxia del habla la articulación de los sonidos es deficiente pero variable: a veces articulan bien un sonido, luego lo hacen en forma distorsionada, o producen quiebres articulatorios (cambian un sonido por otro), lo que contrasta con la regularidad del defecto de las disartrias. El lenguaje expresivo de estos pacientes es poco fluente $y$ presentan dificultad para evocar los nombres y verbos. El defecto más característico es el agramatismo, con mal uso u omisión de gramemas (morfemas con significado gramatical); tienden a usar los verbos en infinitivo y a omitir conjunciones y preposiciones. La comprensión es casi normal, salvo para oraciones complejas (oraciones pasivas o pasivonegativas, por ejemplo "el niño no es perseguido por el perro"), en las que existe agramatismo receptivo. En la mayoría de los casos la lesión compromete el área de Broca, pero para que sea persistente debe existir además un compromiso subcortical ${ }^{17}$. Los pacientes con afasia de Broca tienen noción de 
enfermedad, y no es extraño que muestren ansiedad o depresión ${ }^{18}$.

La afasia de Wernicke o afasia sensorial se caracteriza por ser una afasia fluente, con conservación de prosodia, pero que transmite poca información por la dificultad para evocar sustantivos y por la existencia de parafasias ${ }^{10}$. Estas parafasias pueden ser fonémicas, semánticas, verbales o neologismos afásicos. Los pacientes construyen frases y usan gramemas, pero las parafasias hacen difícil saber si son los adecuados. La comprensión verbal es deficiente, ya sea que se trate de discriminar estructuras gramaticales, palabras aisladas o, en menor medida, fonemas. Las lesiones generalmente comprometen la parte posterior de la circunvolución temporal superior izquierda; el tamaño de la lesión y su extensión a las circunvoluciones angular y supramarginal influyen en el pronóstico ${ }^{19}$. Ocasionalmente se observa con lesiones talámicas izquierdas e hipoperfusión cortical $^{2}$. Con frecuencia los pacientes no tienen noción de enfermedad (anosognosia), y presentan actitudes emocionales anormales, incluso psicóticas. No tienen conciencia de que hablan mal ni de que su comprensión es deficiente, pero sí perciben su fracaso comunicativo -no obtienen lo que desean- y suelen molestarse con el interlocutor.

La sordera pura de palabras, como variante de la afasia de Wernicke, puede verse con lesiones temporales izquierdas pequeñas o bilaterales y puede asociarse a agnosia auditiva $^{19}$ y alexia con afasia.
La afasia de conducción ha sido atribuida clásicamente a una lesión del fascículo arcuato, que impide la repetición al desconectar las áreas de Broca y Wernicke. En los últimos años se ha señalado la importancia de la circunvolución supramarginal, ubicada sobre ese fascículo, en el procesamiento (reconocimiento y selección) de los fonemas ${ }^{19}$. El área de Wernicke conserva sus conexiones con el resto del cerebro a través de la circunvolución angular y la comprensión es normal; pero existe un marcado defecto de la repetición y en el lenguaje expresivo, con parafasias fonémicas y moderada pérdida de fluidez. Es característica la "conducta de aproximación": el paciente se da cuenta de sus errores e intenta corregirlos: "esto es una tesa... nesa... imesa!".

En las afasias transcorticales la repetición está conservada e incluso existe ecolalia (tendencia a repetir lo que dijo el interlocutor). En la afasia transcortical sensorial existe un severo defecto de la comprensión con repetición automática, y el lenguaje expresivo es vacío, semejante al de una afasia de Wernicke, con gran predominio de las parafasias semánticas. Según Crosson $^{11}$ esta afasia sería característica de las lesiones talámicas, pero también puede verse por lesión de las circunvoluciones temporal media e inferior posteriores, con respeto del área de Wernicke ${ }^{19}$. En la afasia transcortical motora existe un severo defecto para formular el discurso, en la capacidad de hablar sobre un tema; en cambio están respetados la denominación, el lenguaje automático, la repetición y la comprensión. En la etapa inicial puede existir mutismo. Este tipo de afasia se debe a lesiones frontales que respetan el 
área de Broca, y es similar a un síndrome frontal con pérdida de iniciativa restringida al lenguaje ${ }^{1,19}$.

La afasia global se debe a lesiones extensas del territorio silviano izquierdo. Existe un severo defecto de la comprensión (comprenden sólo a veces órdenes de un elemento) y de la expresión, que se encuentra reducida a breves emisiones inarticuladas o a estereotipias afásicas y a emisiones de algunos sonidos, por ejemplo “í-illó, illó". Estas estereotipias llegan a adquirir una prosodia que informa sobre el estado anímico del paciente. Al pedirles describir la lámina 1 de la prueba de Boston se limitan a repetir esas mismas sílabas, pero llama la atención que a veces señalan con el dedo cada uno de los detalles importantes de la escena; tienen un gran defecto lingüístico pero son capaces de reconocer los elementos esenciales de una escena, a diferencia de los que suele verse en pacientes con lesiones del hemisferio derecho. En la afasia no fluente mixta también existe un defecto de todos los aspectos del lenguaje pero es de menor cuantía, y el paciente tiene un lenguaje no fluente, con apraxia del habla, parafasias y un defecto importante en la comprensión ${ }^{10}$.

\subsection{Los pacientes afásicos.}

Para referirnos a ellos se deben considerar sus antecedentes clínicos, la actitud frente al defecto, el deterioro intelectual y otros defectos asociados ${ }^{6}$. Las lesiones que causan afasias son fundamentalmente los accidentes cerebrovasculares, los traumatismos cráneo encefálicos y afecciones degenerativas del cerebro. Los accidentes cerebrovasculares generalmente se dan en sujetos mayores de 60 años; se ha calculado que dos de cada tres pacientes con lesiones vasculares del hemisferio izquierdo tienen una afasia, de severidad variable; constituyen la etiología más frecuente entre los sujetos que son sometidos a tratamiento fonoaudiológico. Las afasias traumáticas son menos frecuentes, pero como generalmente se dan en personas jóvenes hay mayor interés por su rehabilitación. En los traumatismos es más frecuente el compromiso de los polos frontales y temporales, por eso son frecuentes las afasias de Wernicke y el mutismo o laconismo (como parte de un síndrome frontal) más que una afasia de Broca o de conducción, que generalmente se deben a lesiones vasculares.

Las afasias en afecciones degenerativas tales como la enfermedad de Alzheimer son cada vez más frecuentes, pero al ser parte de una demencia generalmente no se envían a rehabilitación. Otras causas de afasias -tumores, encefalitis, abscesos cerebrales- son menos frecuentes. Como casos especiales se puede mencionar la afasia progresiva sin demencia descrita por Mesulam, que es una afasia no fluente, y la afasia semántica (fluente); ambas son variantes de las demencias frontotemporales ${ }^{20}$; rara vez se deben a una enfermedad de Alzheimer.

Los pacientes afásicos son mayoritariamente de sexo masculino. En efecto, tanto los accidentes cerebrovasculares como los traumatismos son más frecuentes en los hombres; sólo las afecciones degenerativas afectan más a las mujeres. 
La actitud del paciente es influida por la edad, el tipo de afasia, el deterioro intelectual asociado, y otros factores. Los pacientes de más edad tienen menos plasticidad cerebral para la recuperación del déficit, lo que significa que se recuperan menos, pero tanto ellos como sus familias aceptan más fácilmente las secuelas. En cambio, en los jóvenes, la plasticidad y la capacidad de recuperación es mayor, pero tanto el paciente como su familia tienen una menor tolerancia a las secuelas y les resulta más difícil recobrar el equilibrio emocional después de la lesión cerebral.

Con respecto al tipo de afasia, los pacientes con afasias de Broca generalmente tienen clara noción de su déficit, se angustian y se deprimen, pero colaboran más en la rehabilitación ${ }^{18}$. En cambio los pacientes con afasia de Wernicke son más anosognósicos, no tienen conciencia de sus defectos y se angustian o deprimen menos por ellos. Sin embargo tienen conciencia de su fracaso comunicativo, se molestan al no obtener lo que desean, creen que el interlocutor no les presta atención, y colaboran menos en su tratamiento. Cuando existen cuadros psicóticos, ellos se asocian más la afasia de Wernicke que a la de Broca.

La relación entre el lenguaje y la inteligencia es compleja. La mayoría de los afásicos presentan algún grado de deterioro intelectual, pero es difícil de medir por la afasia y no siempre hay paralelismo entre defecto lingüístico e intelectual. Uno de los instrumentos más usados para medir esta capacidad intelectual son las Matrices Progresivas Coloreadas de Raven, una prueba de inteligencia visuo-espacial que no requiere de lenguaje. El defecto intelectual es mayor en las afasias globales y de Wernicke, situación que podemos atribuir en parte a la lesión cerebral y en parte al hecho de que el lenguaje es un instrumento del pensamiento ${ }^{1,5}$

El conocimiento semántico es un conocimiento de larga data sobre objetos, hechos y conceptos $^{21}$. Constituye una parte muy importante de la capacidad cognitiva, y con respecto al lenguaje se puede evaluar en la denominación, la comprensión de palabras, las asociaciones de palabras y la comprensión de metáforas y del discurso. Después de reconocer un objeto, la denominación implica seleccionar su nombre preciso, una propiedad indisoluble de dicho objeto; el proceso inverso se produce al comprender el nombre. Las asociaciones de palabras implican manejar redes semánticas, que en parte son comunes a los grupos culturales y en parte propias de cada individuo. Finalmente, la comprensión del discurso depende -entre otros factores- del conocimiento semántico. En estudios recientes sobre activación cerebral en sujetos normales, se ha comprobado que la comprensión de palabras requiere de la actividad de la corteza temporal, especialmente a izquierda. Si son herramientas se activa la circunvolución fusiforme medial bilateral; si son rostros, la circunvolución fusiforme lateral; si son animales la circunvolución fusiforme lateral y el surco temporal superior; etc. ${ }^{22}$. Estas observaciones son concordantes con el hecho de que existen afasias amnésicas en que destaca la dificultad para denominar personas, animales, herramientas y tal vez otras categoría, con lesiones de diversas topografías ${ }^{7}$. 
También pueden acompañar a las afasias defectos en la expresión y comprensión de la pantomima. Los primeros se confunden con las apraxias ideatoria e ideomotora. Los defectos en la comprensión de la pantomima son frecuentes en las afasias globales y de Wernicke, en las cuales el intento de comunicarse por medio de gestos es habitualmente ineficaz. Estos defectos pueden estudiarse haciendo pantomimas y pidiendo al paciente que señale el referente adecuado, por ejemplo haciendo el gesto de fumar y pidiéndole que escoja entre los dibujos de un cigarrillo, un vaso, un reloj u otros objetos ${ }^{23}$.

\subsection{Tratamiento de las afasias.}

El tratamiento fundamental de las afasias es el tratamiento fonoaudiológico. Este tiene dos objetivos principales: que el paciente logre satisfacer las necesidades comunicativas de la vida diaria en diferentes ambientes, y que logre una adaptación psicosocial en relación a su alteración comunicativa ${ }^{4}$, 25.

El primer objetivo se puede lograr a través de dos estrategias, la reactivación y la compensación de las funciones alteradas. La estrategia de reactivación o facilitación aprovecha en la primera etapa que las neuronas parcialmente dañadas (o alteradas por un proceso de diasquisis) pueden recuperarse. Luego se beneficia de la plasticidad cerebral, que permite que las funciones deficientes pueden ser asumidas por otras áreas del cerebro, incluso por el hemisferio derecho.

Las estrategias compensatorias se refieren a la comunicación aumentativa y alternativa: el paciente (y los que lo rodean) aprendan a comunicarse mediante gestos, prosodia o elementos auxiliares tales como los cuadernos de comunicación.

El tratamiento de las afasias es complejo, y muchas veces requiere que participen en la evaluación y la terapia otros profesionales: kinesiólogo, psicólogo, terapeuta ocupacional, médico fisiatra, y otros. Además de dirigirse al paciente intenta mejorar el entorno comunicativo, debe dirigirse a quienes lo rodean para corregir interacciones o actitudes inadecuadas y lograr que se restablezca la comunicación con el paciente.

\subsection{Trastornos del discurso.}

El discurso es un acto de habla compuesto de uno o varios enunciados, que tiene unidad temática y que se realiza en un contexto determinado. Como prerrequisitos para el discurso podemos mencionar la disposición a la tarea, la percepción visual, la capacidad ejecutiva y la capacidad lingüística. Esta última implica los sistemas semántico-sintáctico y el sistema fonológico y fonético. Existen varios tipos de discurso, pero entre los más estudiados se encuentra el discurso descriptivo aplicado a la lámina 1 de la prueba de Boston $^{10}$

Así como las afasias comprometen especialmente la forma del lenguaje, los trastornos del discurso comprometen especialmente su contenido y su uso o pragmática ${ }^{5}$. Entre los defectos del contenido destaca la incoherencia, por pérdida de la unidad temática. Los defectos pragmáticos se expresan como inadecuación a las circunstancias: dirigirse a extraños en forma coloquial, defectos en la 
teoría de la mente. Entre las condiciones neurológicas que alteran el discurso pueden citarse el compromiso de conciencia, alteraciones de la afectividad, trastornos perceptuales, demencias, lesiones del hemisferio derecho, y afasias. Nos referiremos sólo a algunas de estas condiciones.

En la etapa inicial de la enfermedad de Alzheimer pueden existir trastornos del discurso en relación con las fallas de memoria (por ejemplo perseveraciones), de la capacidad ejecutiva (defectos de programación y de la coherencia), a veces en relación con trastornos afectivos. A medida que avanza la demencia el discurso oral o escrito se va acortando, aparecen errores lexicales de tipo afásico (anomia y parafasias), las frases se hacen más simples o incompletas, se empobrece el contenido o se introducen elementos irrelevantes ${ }^{24,26}$. La articulación, la prosodia y la gramática se respetan hasta etapas muy avanzadas.

En las demencias frontotemporales existe cierta correlación anátomo-clínica para las alteraciones del lenguaje. Las atrofias dorso-laterales tienden a un discurso lacónico y pobre; las órbitofrontales a un discurso inadecuado e incoherente; y las fronto-mediales al uso de expresiones estereotipadas $^{20}$. Trabajos recientes señalan que los pacientes pueden describir los elementos de una escena sin describir la acción, y hacen hincapié en la pérdida de la conectividad o coherencia global, de la capacidad para organizar el contenido. La pérdida de coherencia se asocia a atrofia frontal dorsolateral derecha ${ }^{27,28}$.
Las lesiones del hemisferio derecho pueden causar defectos de la coherencia, de la interpretación metafórica y de la valoración de los detalles del discurso ${ }^{6,13}$. Estos pacientes pueden ser más fluentes que lo normal, pero con disgresiones. Frente a la lámina 1 de la prueba de Boston pueden ser detallistas, enumerar cada uno de los objetos de la escena, o hacer comentarios irrelevantes. En cambio, ya dijimos que pacientes con afasia global, casi sin lenguaje expresivo, suelen indicar los detalles importantes de la escena. En estudios de la comprensión de metáforas se ha observado que los pacientes afásicos, cuando comprenden algo, comprenden el significado metafórico real; en cambio, pacientes con lesiones del hemisferio derecho pueden interpretar esas expresiones de un modo literal ${ }^{13}$. Ante un relato complejo los pacientes con lesiones derechas no discriminan entre los detalles importantes y los irrelevantes; defecto concordante con su tendencia a las disgresiones. Al estudiar la activación cerebral durante el procesamiento del lenguaje en normales se ha demostrado que cuando se escuchan palabras aisladas se activan más las áreas perisilvianas izquierdas; en cambio, al procesar un discurso se va activando el hemisferio derecho, hasta hacerse predominante cuando se llega a la conclusión, a la síntesis final ${ }^{29}$. Otros estudios en normales han concluido que el hemisferio izquierdo tiene ventajas para procesar frases literales y metáforas familiares o simples; y el hemisferio derecho para frases literales o metáforas no familiares, para relaciones semánticas más remotas. Esto incluye el apreciar la ambigüedad, el humor, las inferencias, los requerimientos indirectos ${ }^{30}$. 
2. Trastornos de la lectoescritura.

Los defectos de la lectoescritura dependen en parte de los defectos del lenguaje oral (en afasias y en defectos del discurso), pero también de defectos perceptuales y práxicos; incluso pueden ser independientes del lenguaje oral, como sucede en las alexias y agrafias puras ${ }^{10,19}$.

Las agrafias pueden ser clasificadas en lingüísticas y motoras ${ }^{31,32}$. Entre las agrafias lingüísticas distinguimos la agrafia fonológica, la agrafia profunda y la agrafia superficial (o lexical); muchas veces se asocian a afasias, pero a veces se presentan aisladas. Sus características dependerían del compromiso de las circunvoluciones supramarginal y angular. La primera sería el sustrato del sistema que permite el manejo de los fonemas; en este caso, transformar cada fonema en el grafema correspondiente, indispensable para escribir logotomas (palabras inventadas, por ejemplo "cotifre"). En las circunvoluciones angular y fusiforme existen sistemas que permiten evocar la imagen visual de la palabra (principalmente en la segunda), indispensable para escribir palabras en que no hay concordancia entre fonemas y grafemas (por ejemplo "alcohol").

En la agrafia superficial, el paciente puede escribir bien si se le dictan logotomas, pero fracasa en palabras de grafía irregular. En las agrafias fonológicas, por lesión de la circunvolución supramarginal, los pacientes son capaces de escribir evocando la imagen visual de la palabra, pero fracasan con los logotomas. En las agrafias profundas, por lesiones extensas del territorio silviano izquierdo, se supone que el paciente escribe en base al hemisferio derecho. Esto podría ser la causa de las paragrafias semánticas, síntoma principal de este cuadro. A estos pacientes le es más fácil la escritura de las palabras concretas que de las abstractas, y de los sustantivos más que de las palabras gramaticales $^{31}$.

La escritura puede alterarse por patologías motoras tales como el parkinsonismo o un síndrome cerebeloso; pero al hablar de agrafias motoras se referiere a agrafias apráxicas y visuoespaciales. En las primeras hay distorsión de los grafemas con o sin perseveración en los trazos, de tal modo que las letras se hacen irreconocibles. Con frecuencia se asocian a apraxias ideomotoras o constructivas, pero pueden disociarse. Las agrafias visuoespaciales se caracterizan por negligencia espacial unilateral, mala disposición espacial de los renglones, separación anormal de palabras o letras ${ }^{32}$. Pueden asociarse a apraxias constructivas.

La clasificación más tradicional de las alexias incluye la alexia sin agrafia, la alexia con agrafia y la alexia frontal. La alexia sin agrafia es un síndrome de desconexión por lesión del polo occipital izquierdo (con hemianopsia homónima derecha) y del esplenio del cuerpo calloso (que desconecta las áreas de asociación visual de uno y otro hemisferio). Esto determina que la información visual llegue sólo al polo occipital derecho. Según Geschwind, si el paciente ve un objeto, este tiene asociaciones somestésicas que pueden transmitirse a las áreas del lenguaje a través de la parte media del cuerpo calloso, y logra denominarlo. En cambio la información sobre letras o colores debería 
transmitirse al hemisferio izquierdo a través del esplenio; y su lesión causa una alexia asociada a anomia de colores $^{33}$. La alexia con agrafia se ha atribuido a una lesión de la circunvolución angular izquierda, y la tercera alexia o alexia frontal, por lesiones del área de Broca, se caracterizaría por la dificultad para leer o denominar letras aisladas y palabras gramaticales.

Desde el punto de vista de la psicología cognitiva las alexias (o dislexias) pueden ser clasificadas en centrales y periféricas. En las últimas existe un defecto del procesamiento visual del estímulo, y en las centrales un defecto en el paso entre la forma visual y el lenguaje o el significado propiamente tal ${ }^{34}$.

Entre las dislexias periféricas se encuentra la alexia sin agrafia ya descrita, la dislexia por negligencia unilateral (cometen errores porque no leen el extremo izquierdo de las palabras o frases), la dislexia atencional (en demencias o compromiso de conciencia), y la hemialexia en el hemicampo visual izquierdo cuando existe una desconexión por agenesia o sección del cuerpo calloso.

Las dislexias centrales son la dislexia superficial, la dislexia fonológica y la dislexia profunda $^{34}$. En la dislexia superficial (que podría ser llamada lexical) existe dificultad para leer palabras de grafía irregular (como Llanquihue, alcohol); se ha descrito especialmente en demencias. Los sujetos con dislexia fonológica, que generalmente no son severas, fracasan en la lectura de logotomas. Las lesiones pueden ser de las circunvoluciones supramarginal o angular. La dislexia profunda se asocia a extensas lesiones del hemisferio izquierdo y se caracteriza por la existencia de errores semánticos (en vez de "castillo" podrían leer "palacio") y la dificultad para leer logotomas y palabras abstractas. Leen mejor las palabras sustantivas y concretas, y se ha supuesto que lo hacen a través del hemisferio derecho.

Finalmente, el estudio de la lectoescritura se extendería a la comprensión de textos y al discurso escrito. Los defectos en la comprensión de textos suelen confundirse con los dependientes de las fallas de atención y memoria. En el discurso escrito los defectos generalmente son similares a los observados en el discurso oral, pero más precoces y severos $^{26}$. Se observa reducción del número de palabras, pérdida de coherencia, anomia y parafasias. Los defectos lexicales son muy sugerentes de enfermedad de Alzheimer.

\section{Comentario Final}

Hace pocos años un conocido texto de neurología decía "las alteraciones cerebrales del lenguaje son las afasias". Esperamos haber aclarado que si bien las afasias son las más conocidas y frecuentes, existen muchos otros trastornos que deben ser detectados por el neurólogo y el fonoaudiólogo clínico. El desarrollo de la imagenología ha reducido el valor de estos defectos en la localización de las lesiones cerebrales, pero su evaluación es indispensable para el manejo del paciente, y de gran importancia para su rehabilitación. 


\section{Referencias}

1. Luria A, (1980). Higher cortical functions in man. 2a. ed, Basic Books, New York.

2. Metter EJ. (1987). Neuroanatomy and physiology of aphasia: evidence from positron emission tomography. Aphasiology; 1:3-34.

3. Donoso A, Faure E y Vejar MA. (1986). Trastornos neuropsicológicos en lesiones talámicas. Rev Chil Neuro-Psiquiat; 24:47-52.

4. Chapey R. (2001). Language intervention strategies in aphasia and related neurogenic communication disorders. 4o ed. Philadelphia, Lippincott Williams \& Wilkins.

5. Bloom L y Lahey M. (1978). Language development and language disorders. Wiley \& Sons, New York.

6. Donoso A. (1999). Cerebro y lenguaje. Ed. Universitaria, Santiago.

7. Damasio H, Granel D, Grawobski T, Adolphs R, Damasio A. (2004). Neural systems behind word and concept retrieval. Cognition; 92:179-229.

8. Dronkers NF. (1996). A new brain region for coordinating speech articulation. Nature; 384:159-61.

9. Lhermitte F y Gauthier JC. (1969). Aphasia. En: Handbook of Clinical Neurology, vol 4. PJ Vinken y GW Bruyn eds, North Holland Publ Co, Amsterdam.

10. Goodglass H y Kaplan E. (1986). Evaluación de la afasia y trastornos similares, 2ª. Ed. Panamericana, Madrid.

11. Crosson B. (1985). Subcortical functions in language: a working model. Brain \& Language; 25:257-92.

12. Donoso A, Pavez M y Santander M. (1981). Hemisferectomía izquierda en un adulto diestro. Rev Neurol (Barcelona); 9:345-52.

13. Wapner W, Hamby S y Gardner H. (1981). The role of the right hemisphere in the apprehension of complex linguistic materials. Brain \& Language; 14:15-33.

14. Kaczmareck B. (1987). Regulatory functions of the frontal lobes: a neurolinguistic perspective. En: The frontal lobes revisited, E Perecman ed, The IBN Press, New York.

15. Basso A, Lecours AR, Morschini S y Vanier M. (1985). Anatomoclinical correlations in the aphasias as defined through computarized tomography: exceptions. Brain \& Language; 26:201-29.

16. Cuetos F: ¿Por qué cometen errores semánticos los pacientes afásicos? www.uninet.edu/union99/congress/confs/lang/01Cuet os.html.

17. Mohr J, Pessin MS, Finkelstein S y cols. (1978). Broca's aphasia: pathological and clinical aspects. Neurology; 28:311-24.

18. Donoso A, Santander M, Pavez M y cols. (1982). Evaluación multiprofesional de las afasias en pacientes hospitalizados. Rev Med Chile; 110:249-54.

19. Alexander MP. (1997). Aphasia: clinical and anatomical aspects. En: T Feinberg y M Farah eds: Behavioral neurology and neuropsychology, McGraw-Hill, New York.

20. Snowden J, Neary D y Mann D. (1996). Fronto-temporal lobar degeneration, Churchill Livingstone, New York.

21. Levy DA, Bayley PJ, Squire LR. (2004). The anatomy of semantic knowledge: medial vs. lateral temporal lobe. PNAS; 101:6710-6715.

22. Caramazza A, Mahon BZ. (2005). The organisation of conceptual knowledge in the brain: the future's past and some future directions. Cogn Neuropsychol; 22:125.

23. González R, Toledo L, Helo A y Donoso A. (2005). Expresión de la pantomima en pacientes afásicos. Rev Chil Fonoaudiología; 6:31-40.

24. González R, Donoso A. (2000). Programa de rehabilitación fonoaudiológica para pacientes afásicos. Rev Chil Fonoaudiología; 2:35-48.

25. Croisile B, Ska B, Brabant MJ, Duchenne A, Lepage $Y$, Aimarg G, Trillet M. (1996). Comparative study of oral and written picture desscription in patients with Alzheimer's disease. Brain \& Language; 53:1-19. 
26. Donoso A, González R. (1995). Lenguaje oral y escrito en demencia de tipo Alzheimer. Rev Chil NeuroPsiquiat; 33:51-56.

27. Ash S, Moore P, Antani S, McCawley G, Work M, Grossman M. (2006). Trying to tell a tale: discourse impairments in progressive aplasia and frontotemporal dementia. Neurology; 66(9): 1405-13.

28. Donoso A, Arecheta P y González R. (2009). Lenguaje oral en demencias frontotemporales, experiencia personal y revisión del tema. Rev Chil Neuro-Psiquiat; 47:114-123.

29. Xu J, Kemeny S, Park G, Frattali K, Braun A. (2005). Language in context: emergent features of word, sentence and narrative comprensión. Neuroimage; 25:1002-1015.

30. Schmidt GL, DeBuse CJ, Seger CA. (2007). Right hemisphere metaphor processing? Characterizing the lateralization of semantic processes. Brain \& Language; 100:127-141.

31. Roeltgen D. (1985). Agraphia. En: Clinical neuropsychology, 2a. ed, K Heilman y E Valenstein eds, Oxford University Press, New York.

32. Donoso A y González R. (1987). Alteraciones neuropsicológicas de la escritura. Rev Chile NeuroPsiquiat; 25:59-62.

33. Geschwind N. (1965). Disconnection syndromes in animals and man. Brain; 88:237-294 y 585-644.

34. Coslett HB. (2003). Acquired disorders of reading. En: Behavioral neurology and neuropsychology, 2a ed. TE Feinberg y M Farah eds., McGraw-Hill, New York. 